\title{
The Effects of Bazedoxifene on Bone, Glucose, and Lipid Metabolism in Postmenopausal Women With Type 2 Diabetes: An Exploratory Pilot Study
}

\author{
Taishi Yoshii ${ }^{a}$, , Masayo Yamada ${ }^{a}$, Taichi Minami ${ }^{a}$, Tetsuji Tsunoda ${ }^{a}$, Mayuko Sasaki ${ }^{a}$, \\ Yoshinobu Kondo ${ }^{c}$, Shinobu Satoh ${ }^{c}$, Yasuo Terauchib, d
}

\begin{abstract}
Background: Selective estrogen receptor modulators (SERMs) decrease homocysteine and cross-linking of pentosidine and reduce low-density lipoprotein cholesterol (LDL-C), and they are expected to improve bone quality and atherosclerosis. Therefore, the potential effects of bazedoxifene on bone (bone resorption, bone formation, and bone quality), as well as on glucose and lipid metabolism markers, were examined in Japanese postmenopausal women with type 2 diabetes mellitus (T2DM).
\end{abstract}

Methods: Eligible patients received $20 \mathrm{mg}$ of bazedoxifene tablets once daily and were followed up for 12 weeks. Bone resorption markers including tartrate-resistant acid phosphatase 5b (TRACP-5b), bone formation markers and bone quality markers such as homocysteine and serum pentosidine, total cholesterol (TC), LDL-C, high-density lipoprotein cholesterol (HDL-C), triglycerides (TG), and HbAlc were all measured.

Results: Twenty patients completed this study. All bone resorption markers decreased significantly 4 weeks after bazedoxifene treatment. In particular, TRACP-5b decreased significantly at 12 weeks (median percent change: $-20.6 \%$ ), and the minimum significant change (MSC) achievement rate of TRACP-5b was $65 \%$. Bazedoxifene also decreased bone formation markers. However, bazedoxifene did not improve bone quality markers. LDL-C, HDL-C, and non-HDL-C were decreased, but TG was unchanged. Glucose metabolism was

Manuscript accepted for publication July 28, 2015

aDivision of Metabolism and Endocrinology, Department of Internal Medicine, Yokohama Sakae Kyosai Hospital, Federation of National Public Service Personnel Mutual Associations Yokohama, 132 Katsura-cho, Sakae-ku, Yokohama, Kanagawa 247-8581, Japan

bDepartment of Endocrinology and Metabolism, Graduate School of Medicine, Yokohama City University, 3-9 Fukuura, Kanazawa-ku, Yokohama, Kanagawa 236-0004, Japan

'Department of Endocrinology and Metabolism, Chigasaki Municipal Hospital, 5-15-1 Motomura, Chigasaki, Kanagawa 253-0042, Japan

${ }^{\mathrm{d}}$ Corresponding Author: Yasuo Terauchi, Department of Endocrinology and Metabolism, Graduate School of Medicine, Yokohama City University, 3-9 Fukuura, Kanazawa-ku, Yokohama, Kanagawa 236-0004, Japan.

Email: terauchi@yokohama-cu.ac.jp

doi: http://dx.doi.org/10.14740/jocmr2278w not changed after bazedoxifene treatment. In a subgroup analysis, the group of patients in whom the percent change in TRACP-5b exceeded the MSC had no change in pentosidine levels at 12 weeks. However, in the group of patients in whom the percent change in TRACP-5b did not exceed the MSC, pentosidine levels tended to increase.

Conclusions: Bazedoxifene may improve bone resorption markers and LDL-C without affecting glucose metabolism in Japanese postmenopausal women with T2DM.

Keywords: Bazedoxifene; Type 2 diabetes mellitus; Bone metabolism; Selective estrogen receptor modulators; Postmenopausal women

\section{Introduction}

Persistent hyperglycemia in patients with diabetes mellitus is said to be associated with various complications. Bone fragility is one of these diabetic complications that have attracted attention. Insulin deficiency impairs osteoblast formation and function, and as a result, insulin deficiency is associated with decreased bone mass $[1,2]$. Therefore, bone mineral density (BMD) in patients with type 1 diabetes mellitus (T1DM) is lower than in those with type 2 diabetes mellitus (T2DM). However, despite BMD not being low in patients with T2DM, a higher risk of fractures has been reported [3].

Bone strength is determined by BMD and bone quality, and the high risk of fracture in T2DM is thought to be due to reduced bone quality [4]. Increased homocysteine and pentosidine accumulation, advanced glycation end products (AGEs), leads to an increase in non-enzymatic cross-linking in bone, thus reducing bone quality [5-7]. The mechanism by which homocysteine and pentosidine increase in diabetes is thought to be as follows. First, excessive gluconeogenesis due to hyperglycemia causes a deficiency in vitamin B6. As a result, this vitamin B6 deficiency leads to higher homocysteine levels $[8,9]$. Then, increased glycation and increased oxidative stress due to persistent hyperglycemia promote the formation of pentosidine cross-linking in bone [10].

Selective estrogen receptor modulators (SERMs) have estrogen-like effects that reduce bone resorption and increase BMD. SERMs have also been reported to have antioxidant effects [11]. These antioxidant effects of SERMs may decrease 
homocysteine levels $[12,13]$. In addition, in a rabbit model of reduced bone quality, the antioxidant effects of SERMs decreased pentosidine accumulation [14]. Thus, SERMs are promising drugs to improve bone quality. SERMs may also be beneficial in patients with diabetes mellitus with increased homocysteine and pentosidine accumulation. Moreover, SERMs do not affect glucose metabolism [15], but their specific estrogen-like effect improves lipid metabolism [16]. Therefore, SERMs may also be effective in reducing the progression of arteriosclerosis in patients with diabetes mellitus.

Bazedoxifene is a novel third-generation SERM for the treatment of postmenopausal osteoporosis. Bazedoxifene, compared to raloxifene, reduced the risk of non-vertebral fractures in a high-risk fracture group [17]. Therefore, bazedoxifene may be an effective option for treatment of postmenopausal osteoporosis. However, to the best of our knowledge, the effects of bazedoxifene on bone, glucose, and lipid metabolism in postmenopausal patients with T2DM have not been reported.

The primary purpose of this pilot study was to explore the potential effects of bazedoxifene on bone metabolism in Japanese postmenopausal patients with T2DM. In addition, the effects of bazedoxifene on glucose and lipid metabolism markers and on indices of arteriosclerosis were also investigated.

\section{Materials and Methods}

\section{Study protocol/patients}

This pilot study was performed as a multicenter, prospective, observational study between February 2013 and September 2013 at three institutes in Japan. This study was approved by the ethics committees of each participating site and it was performed in accordance with the Declaration of Helsinki. This study was registered with the UMIN Clinical Trial Registry (UMIN 000010074).

Patients with T2DM who regularly visited the hospital were assessed for eligibility for this study. Inclusion criteria were as follows: 1) $>2$ years since menopause; 2 ) $<85$ years of age; 3 ) no fluctuations in HbAlc levels $>0.5 \%$ in the past 3 months; 4) HbA1c levels $<8.4 \%$ over the past 3 months; 5) body mass index (BMI) $<30 \mathrm{~kg} / \mathrm{m}^{2} ; 6$ ) estimated glomerular filtration rate (eGFR) levels $>30 \mathrm{~mL} / \mathrm{min} / 1.73 \mathrm{~m}^{2} ; 7$ ) no requirement for new or changed diabetes drug regimens within 4 weeks of starting bazedoxifene treatment; and 8) no requirement for new or changed angiotensin II receptor antagonist, statin, or proton pump inhibitor regimens within 4 weeks of starting bazedoxifene treatment.

Exclusion criteria were as follows: 1) previous history of non-traumatic bone fracture; 2) thiazolidine medication; 3) steroid hormone medication; 4) oral medication for abnormal thyroid function; 5) other medications, including active vitamin $\mathrm{D}_{3}$ drugs, vitamin $\mathrm{K}_{2}$, raloxifene, bisphosphonate, denosumab, or parathyroid hormone (PTH) treatment for osteoporosis; 6) history of deep vein thrombosis; or 7) the presence of any malignancies.

In the present study, the baseline BMD value was not included in the eligibility criteria because T2DM patients are at high risk for fracture due to reduced bone quality despite BMD not being low. Therefore, patients were recruited regardless of the baseline BMD value. All patients provided their written informed consent prior to participation in the study.

Eligible patients received $20 \mathrm{mg}$ of bazedoxifene tablets once daily and were followed up for 12 weeks. During the 12week study period, no changes or additions to drugs for treatment of diabetes mellitus, hypertension, or lipid metabolism were permitted. In addition, no additional drugs for treatment of osteoporosis were allowed.

Primary endpoints were changes in the bone metabolism markers, including bone resorption markers (urinary type I collagen cross-linked N-telopeptide (u-NTX), urinary type I collagen cross-linked C-telopeptide (u-CTX), and tartrate-resistant acid phosphatase 5b (TRACP-5b)), bone formation markers (osteocalcin (OC) and undercarboxylated osteocalcin (ucOC)), and bone quality markers (homocysteine (HC) and serum pentosidine (PS)). Secondary endpoints were changes in $\mathrm{HbAlc}$, high molecular weight (HMW) adiponectin, and the lipid metabolism markers (total cholesterol (TC), low-density lipoprotein cholesterol (LDL-C), high-density lipoprotein cholesterol (HDL-C), and triglycerides (TG)).

\section{Assessments}

To evaluate the patients' condition at baseline (week 0), lumbar spine and femoral neck bone mineral densities were assessed using T-scores and young adult mean (YAM) values from dual-energy X-ray absorptiometry (Discovery X, HOLOGIC), and the fracture risk assessment tool $\left(\right.$ FRAX $\left.^{\circledR}\right)$ created by the World Health Organization was used as an index of the risk for major osteoporotic and hip fractures within the next 10 years.

Overnight fasting blood and urine samples were collected, and weight was measured at 0,4 , and 12 weeks after bazedoxifene treatment.

To evaluate bone metabolism, the bone resorption, formation, and quality markers were measured at the central research center of SRL (SRL, Inc., Tokyo, Japan).

The minimum significant change (MSC) of the bone resorption markers was used for measuring the effect of bazedoxifene. The MSC is used for measuring the effects of osteoporosis therapies. The MSC of u-NTX, u-CTX, and TRACP-5b has been defined as $27.3 \%, 23.5 \%$, and $12.4 \%$, respectively $[18,19]$. When the percent changes of bone resorption markers after treatments were over the MSC, the osteoporosis therapy was considered effective.

To evaluate glucose metabolism, fasting plasma glucose (FPG) (by the glucose oxidation method, chemical reagent and Glucose AUTO, and STAT GA-1160 analyzer; Arkray Inc., Kyoto, Japan), fasting insulin (SRL, Inc.), fasting plasma C-peptide (SRL, Inc.), HbA1c levels (Adams A1c HA-8160, Arkray Inc.), and HMW adiponectin (SRL, Inc.) were measured at 0,4 , and 12 weeks. HOMA-IR and HOMA- $\beta$ represent insulin resistance and pancreatic $\beta$-cell function, respectively, and were calculated as follows: HOMA-IR = fasting insulin $(\mu \mathrm{U} / \mathrm{mL}) \times$ fasting plasma glucose $(\mathrm{mg} / \mathrm{dL}) / 22.5$, and HOMA- $\beta=20 \times$ fasting insulin $(\mu \mathrm{U} / \mathrm{mL}) /($ fasting plasma glucose $(\mathrm{mg} / \mathrm{dL})-3.5)$. 
Table 1. Patients' Baseline Characteristics

\begin{tabular}{|c|c|}
\hline & Baseline $(n=20)$ \\
\hline Age (years) & $67.9 \pm 5.5$ \\
\hline Menopause age (years) & $50.2 \pm 5.1$ \\
\hline Diabetes duration (years) & $4.5(1-24)$ \\
\hline Height (cm) & $153.5 \pm 4.4$ \\
\hline BMI $\left(\mathrm{kg} / \mathrm{m}^{2}\right)$ & $22.5 \pm 2.8$ \\
\hline HbAlc (\%) & $6.3(5.4-7.4)$ \\
\hline Serum creatinine (mg/dL) & $0.61(0.39-0.82)$ \\
\hline \multicolumn{2}{|l|}{ Microangiopathy } \\
\hline Neuropathy (\%) & $29(5 / 17)$ \\
\hline Retinopathy (\%) & $0(0 / 17)$ \\
\hline \multicolumn{2}{|l|}{ Nephropathy (\%) } \\
\hline Stage 1 & $82(14 / 17)$ \\
\hline Stage 2 & $18(3 / 17)$ \\
\hline \multicolumn{2}{|l|}{ Macroangiopathy } \\
\hline Coronary artery disease (\%) & $5.9(1 / 17)$ \\
\hline Cerebrovascular disease (\%) & $24(4 / 17)$ \\
\hline Peripheral artery disease (\%) & $0(0 / 17)$ \\
\hline \multicolumn{2}{|l|}{ Smoking $(\%)$} \\
\hline Former smoker & $20(5 / 20)$ \\
\hline Current smoker & $0(0 / 20)$ \\
\hline Alcohol (\%) & $0(0 / 20)$ \\
\hline \multicolumn{2}{|l|}{ Lumbar } \\
\hline Bone mineral density $\left(\mathrm{g} / \mathrm{cm}^{2}\right)$ & $0.9 \pm 0.2$ \\
\hline T-score & $-1.3 \pm 1.6$ \\
\hline YAM (\%) & $86.4 \pm 17.0$ \\
\hline \multicolumn{2}{|l|}{ Femoral neck } \\
\hline Bone mineral density $\left(\mathrm{g} / \mathrm{cm}^{2}\right)$ & $0.6 \pm 0.1$ \\
\hline T-score & $-1.6 \pm 0.8$ \\
\hline YAM (\%) & $78.2 \pm 10.9$ \\
\hline \multicolumn{2}{|l|}{ FRAX $^{\circledR}$ tool } \\
\hline Major osteoporotic (years) & $8.3(5.2-24)$ \\
\hline Hip fracture (years) & $1.3(0.4-7.6)$ \\
\hline History of the vertebral fracture & $0(0 / 20)$ \\
\hline History of the non-vertebral fracture & $0(0 / 20)$ \\
\hline Family history of the femoral neck fracture & $0(0 / 20)$ \\
\hline \multicolumn{2}{|l|}{ Medications } \\
\hline \multicolumn{2}{|l|}{ Anti-diabetes } \\
\hline Diet only (\%) & $15(3 / 20)$ \\
\hline Sulfonylureas (\%) & $20(4 / 20)$ \\
\hline Glinides $(\%)$ & $10(2 / 20)$ \\
\hline Biguanides $(\%)$ & $25(5 / 20)$ \\
\hline DPP-IV inhibitors (\%) & $45(9 / 20)$ \\
\hline GLP-1 analogues $(\%)$ & $10(2 / 20)$ \\
\hline
\end{tabular}

Table 1. Patients' Baseline Characteristics - (Continued)

\begin{tabular}{cl}
\hline & Baseline $(\mathbf{n}=\mathbf{2 0})$ \\
\hline$\alpha$-glucosidase inhibitors (\%) & $5(1 / 20)$ \\
Insulin (\%) & $5(1 / 20)$ \\
Anti-dyslipidemia & \\
Stains (\%) & $35(7 / 20)$ \\
Ezetimibe (\%) & $15(3 / 20)$ \\
Anti-hypertension & \\
ARBs (\%) & $30(6 / 20)$ \\
\hline
\end{tabular}

Data are reported as means \pm standard deviation or medians (min max). BMI: body mass index; YAM: young adult mean; FRAX ${ }^{\circledR}$ tool: Fracture Risk Assessment Tool; ARBs: angiotensin II receptor blocker.

To evaluate lipid metabolism, TC, LDL-C, HDL-C, and TG (Kyowa Medex, Tokyo, Japan) were measured at 0, 4, and 12 weeks. Non-HDL-C (mg/dL) was calculated as follows: TC (mg/dL) - HDL-C (mg/dL).

The levels of other markers, aspartate amino transaminase (AST), alanine amino transaminase (ALT), gamma-glutamyl transpeptidase $(\gamma \mathrm{GTP})$, and serum creatinine $(\mathrm{Cr})$, were measured at 0,4 , and 12 weeks. The urinary albumin-to-creatinine ratio (UACR) and cystatin $\mathrm{C}$ levels were measured at 0 and 12 weeks. Brachial ankle pulse wave velocity (baPWV) (VaSera VS-1000, Fukuda Denshi), high sensitivity C-reactive protein (hs-CRP), and ferritin levels were measured as arteriosclerosis indices at 0 and 12 weeks.

\section{Adverse events}

Safety was assessed on the basis of all reported adverse events identified by the physicians' examinations and blood parameters.

\section{Statistical analysis}

Statistical analysis was conducted using the Statistical Package for the Social Sciences (SPSS version 11.0.1 J, SPSS Inc., Armonk, NY).

Baseline characteristics are summarized descriptively. To investigate the effects of the intervention, the median value was calculated at each point, and the change from baseline was tested using the Wilcoxon signed-rank test at each post-treatment point. Median percent change from baseline was also calculated. In addition, the MSC achievement rates were computed as the proportion of patients who achieved an improvement exceeding the MSC for each bone resorption marker. Moreover, subgroup analyses were conducted to explore the effects of bazedoxifene on bone, glucose, and lipid metabolism markers based on glycemic control at baseline and whether they exceeded the MSC of TRACP-5b at 12 weeks. For the analysis of HOMA-IR and HOMA- $\beta$, patients on insulin therapy were excluded.

The level of statistical significance was set at 0.05 . Due to the exploratory nature of the study, no correction for multiple 
Table 2. Values of All Clinical Parameters From 0 to 12 Weeks

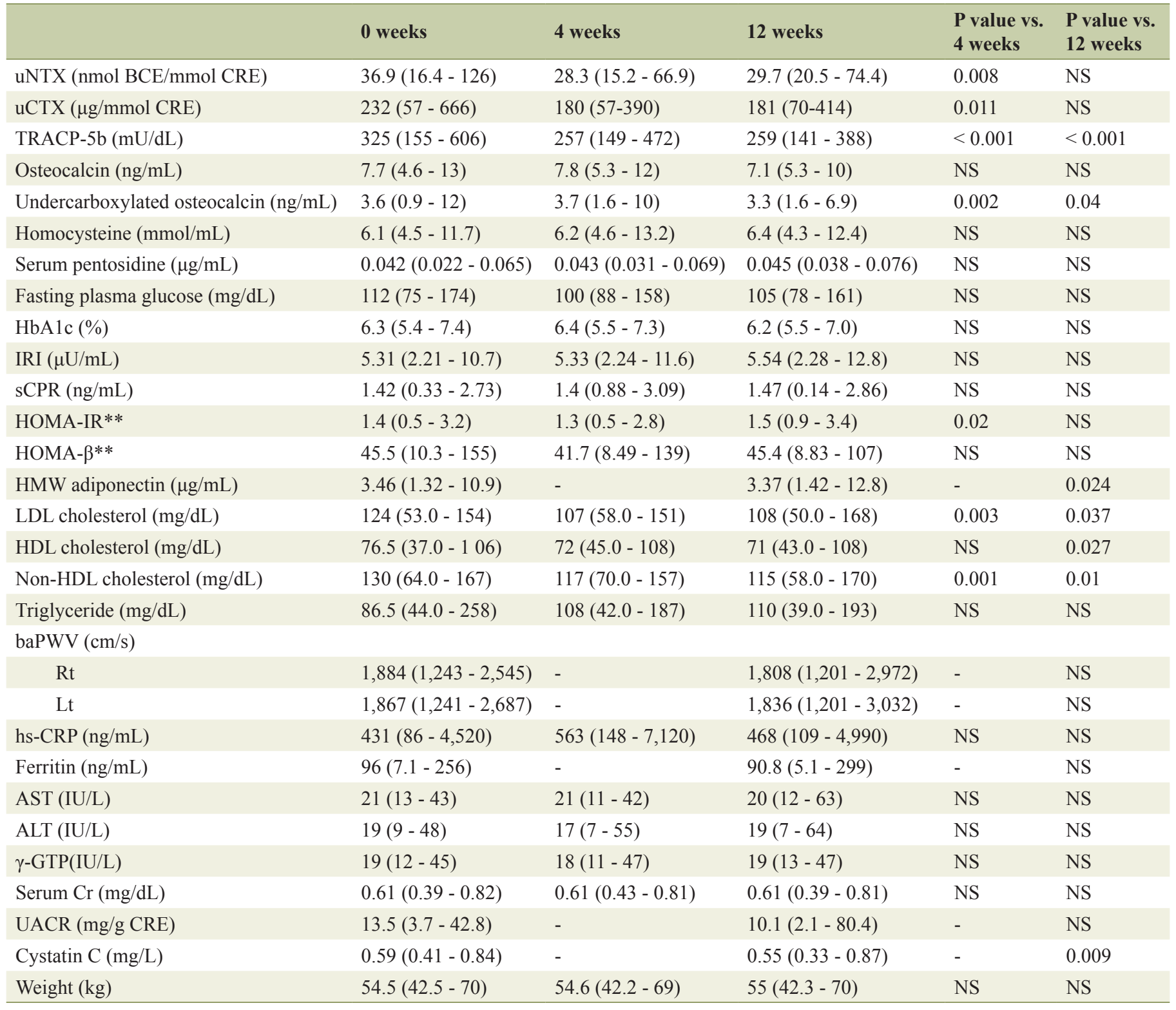

*Data are reported as medians (min - max). ${ }^{*}$ Patients with insulin therapy $(n=1)$ were excluded from analysis of HOMA-IR and HOMA- $\beta$. UACR: urinary albumin-to-creatinine ratio; NS: no significance.

significance testing was made.

\section{Results}

Of the 23 patients enrolled, 20 completed this study. Two patients withdrew from this trial before starting the administration of bazedoxifene; one was found to be ineligible after enrollment, and the other withdrew consent. One patient was withdrawn after the administration of bazedoxifene because of suspected angina pectoris.

The patients' baseline characteristics are shown in Table 1. The mean age ( \pm standard deviation) was $67.9 \pm 5.5$ years, and the mean BMI was $22.5 \pm 2.8 \mathrm{~kg} / \mathrm{m}^{2}$. These patients were not obese. All patients had good glucose control (median HbA1c $6.3 \%$ ) and short diabetes duration (median 4.5 years) in the present study. Femoral neck BMD was moderately decreased, but lumbar BMD was normal. All patients had no clinical and morphometric fractures.

All clinical parameters, such as bone, glucose, lipid, and other levels, are shown in Table 2.

\section{Bone resorption markers}

The levels of u-NTX, u-CTX, and TRACP-5b decreased sig- 


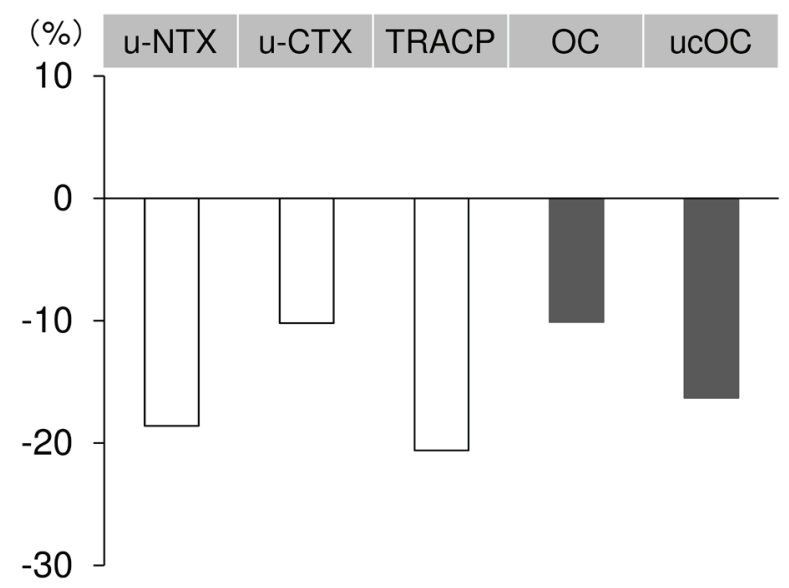

Figure 1. Median percent changes in bone metabolism after 12 weeks of bazedoxifene treatment. Median values (first quartile, third quartile) are $-18.6 \%(-30.9 \%, 12.9 \%)$ for u-NTX, $-10.2 \%(-44.1 \%, 20.7 \%)$ for u-CTX, $-20.6 \%(-27.8 \%,-5.8 \%)$ for TRACP, $-10.1 \%(-17.7 \%, 8.6 \%)$ for OC, and $-16.3 \%(-23.4 \%,-2.2 \%)$ for ucOC. u-NTX: urinary type I collagen cross-linked N-telopeptide; u-CTX: urinary type I collagen crosslinked C-telopeptide; TRACP: tartrate-resistant acid phosphatase 5b; OC: osteocalcin; ucOC: undercarboxylated osteocalcin.

nificantly from baseline after 4 weeks of bazedoxifene treatment $(\mathrm{P}=0.008, \mathrm{P}=0.011$, and $\mathrm{P}<0.001$, respectively). In particular, TRACP-5b levels decreased significantly at 12 weeks.

Median percent changes in bone resorption and formation makers after 12 weeks of treatment with bazedoxifene are shown in Figure 1. The median percent changes of u-NTX, $\mathrm{u}-\mathrm{CTX}$, and TRACP-5b at 12 weeks were $-18.6 \%,-10.2 \%$, and $-20.6 \%$, respectively (Fig. 1). The median percent change exceeded the MSC only for TRACP-5b.

The MSC achievement rate at 12 weeks was the highest for TRACP-5b (65\%), followed by u-CTX (45\%) and u-NTX $(30 \%)$.

\section{Bone formation markers}

The OC levels remained stable over the 12 weeks, but the ucOC levels were significantly decreased at 12 weeks $(\mathrm{P}=0.040)$.

\section{Bone quality markers}

Homocysteine and serum pentosidine levels were not changed after administration of bazedoxifene.

\section{Lipid metabolism}

Bazedoxifene significantly reduced LDL-C levels from 124 (53.0 - 154) (median, (min - max)) $\mathrm{mg} / \mathrm{dL}$ at baseline to 107 $(58.0-151) \mathrm{mg} / \mathrm{dL}$ and $108(50.0-168) \mathrm{mg} / \mathrm{dL}$ at 4 and 12 weeks $(\mathrm{P}=0.003, \mathrm{P}=0.037)$, respectively. The LDL-C median percent changes from baseline were $-11.6 \%$ and $-6.2 \%$ at 4 and
12 weeks, respectively. HDL-C levels and non-HDL-C levels decreased from $76.5(37.0$ - 106) $\mathrm{mg} / \mathrm{dL}$ and 130 (64.0 - 167) $\mathrm{mg} / \mathrm{dL}$ at baseline to $71.0(43.0-108) \mathrm{mg} / \mathrm{dL}$ and 115 (58.0 $170) \mathrm{mg} / \mathrm{dL}$ at 12 weeks $(\mathrm{P}=0.027, \mathrm{P}=0.010)$, respectively. TG levels were unchanged.

\section{Glucose metabolism}

HbA1c, FPG, HOMA-IR, and HOMA- $\beta$ were relatively stable over the 12 weeks. However, HMW adiponectin levels decreased significantly at 12 weeks $(\mathrm{P}=0.024)$.

\section{Other markers}

The indices of atherosclerosis, baPWV (Rt/Lt), hs-CRP, and ferritin were unchanged. In addition, AST, ALT, and $\gamma$-GTP levels did not change after bazedoxifene treatment. Serum creatinine levels were also stable, but cystatin C levels were significantly decreased at 12 weeks.

\section{Adverse events}

During the study period, no serious adverse events were reported. One suspected case of angina pectoris was observed using a Masters two-step test, but no stenosis was seen on coronary angiography. Other adverse events reportedly related to bazedoxifene treatment such as thrombosis, hepatic dysfunction, or hot flushes were not observed.

\section{Subgroup analyses}

In a subgroup analysis of the effects of bazedoxifene based on glycemic control at baseline, the median percent changes in TRACP-5b at 12 weeks were $-18.0 \%$ in the $\mathrm{HbA} 1 \mathrm{c}<6.5 \%$ group $(n=11)$ and $-25.3 \%$ in the HbA1c $\geq 6.5 \%$ group $(n=9)$. Both groups did not have the statistical significant difference.

Additionally, in a subgroup analysis based on the TRACP$5 \mathrm{~b}$ reduction effect of bazedoxifene, the pentosidine levels were stable in the group of patients $(n=13)$ in whom the percent change in TRACP-5b exceeded the MSC (from $0.043 \mu \mathrm{g}$ ) $\mathrm{mL}$ at baseline to $0.044 \mu \mathrm{g} / \mathrm{mL}$ at 12 weeks). However, the pentosidine level tended to increase in the group of patients (n $=7$ ) in whom the percent change in TRACP-5b did not exceed the MSC (from $0.034 \mu \mathrm{g} / \mathrm{mL}$ at baseline to $0.050 \mu \mathrm{g} / \mathrm{mL}$ at 12 weeks).

\section{Discussion}

The present study is the first to investigate the effects of bazedoxifene in postmenopausal patients with T2DM in an exploratory manner. In this study of Japanese postmenopausal patients with T2DM, bazedoxifene improved bone turnover and reduced LDL-C without affecting glucose metabolism. 
After starting bazedoxifene, all bone resorption markers (u-NTX, u-CTX, and TRACP-5b) decreased after 4 weeks and remained low from week 4 to week 12; the changes from baseline to 4 weeks were significant. In particular, TRACP$5 \mathrm{~b}$ decreased significantly after 12 weeks of treatment with bazedoxifene. TRACP-5b is a serum bone resorption marker reflecting osteoclast activity. TRACP-5b, compared to other bone resorption markers, has less diurnal variation, and it is not affected by renal function or diet [20]. Therefore, TRACP$5 \mathrm{~b}$ is thought to be useful to diagnose osteoporosis and assess the effectiveness of treatment. The MSC for percent improvement in TRACP-5b is reported to be $12.4 \%$ [19].

In the present study, the percent improvement in TRACP5 b following treatment with bazedoxifene was $20.6 \%$, with an MSC achievement rate of $65 \%$, thus showing that bazedoxifene improved bone resorption in patients with T2DM, as well. In a subgroup analysis of the effects of bazedoxifene based on glycemic control before treatment, the median percent changes in TRACP-5b at 12 weeks of treatment with bazedoxifene were $-18.0 \%$ in the HbAlc $<6.5 \%$ group and $-25.3 \%$ in the HbAlc $\geq 6.5 \%$ group. This might suggest that bazedoxifene is effective in improving bone resorption in diabetic patients with poor glycemic control, but the mechanism leading to the observed change remains unclear, and further examinations are needed.

Bazedoxifene decreased bone formation markers in this study. These results are similar to previously reported results. This is thought to be because these bone formation markers, such as OC and ucOC, decreased secondary to the reduction in bone resorption by bazedoxifene.

Bazedoxifene did not decrease bone quality markers such as homocysteine or serum pentosidine in this study. High levels of homocysteine and pentosidine have been reported to be clinical risk factors for fractures. In the Rotterdam study, the relative risk of all fractures with 1-SD higher log homocysteine levels increased by 1.4 (95\% confidence interval $1.2-1.6)$ [21]. High urinary pentosidine levels were an independent predictive factor for new fracture development in postmenopausal women [22]. Serum pentosidine levels are also associated with existing fractures, independent of age and BMD, in postmenopausal patients with T2DM [6].

With regard to the bone quality improvement effects of SERMs, raloxifene has been reported to decrease homocysteine levels and to decrease pentosidine cross-linking [12, 14]. However, no consensus has yet been reached on the mechanism of improvement. Moreover, improvement of serum and urinary pentosidine levels with SERMs has not been clinically reported [23]. Therefore, whether there were any differences in the changes of pentosidine levels associated with the TRACP$5 \mathrm{~b}$ reduction effects of bazedoxifene was also examined. With treatment with bazedoxifene, the group of patients in whom the percent change in TRACP-5b exceeded the MSC had no change in pentosidine levels at 12 weeks. However, in the group of patients in whom the percent change in TRACP-5b did not exceed the MSC, pentosidine levels increased. These findings suggest that greater TRACP-5b reduction effects of bazedoxifene may inhibit an increase in pentosidine levels.

In the present study, the reason for the stable bone quality markers was probably that homocysteine and serum pentosidine levels at baseline were lower in the study patients than in previous reports. The median homocysteine concentration in the present study was $6.1 \mathrm{nmol} / \mathrm{L}$, which is not hyperhomocysteinemia (cut-off level $15 \mathrm{nmol} / \mathrm{L}$ ). In addition, AGEs such as pentosidine are known to be higher in diabetic patients with poor glycemic control and longer diabetes duration [24]. Because the present study patients had relatively good glycemic control and a shorter duration of diabetes, there was probably not enough accumulation of AGEs. Therefore, it was thought that baseline levels of homocysteine and serum pentosidine were too low to observe the improvement with bazedoxifene.

With respect to lipid metabolism, raloxifene and bazedoxifene have previously been reported to decrease LDL-C, but no consensus has been achieved about the effects on HDL-C or TG $[25,26]$. HDL-C is reported to increase with raloxifene and bazedoxifene $[12,17]$. In the present study, bazedoxifene decreased both LDL-C and HDL-C levels. However, bazedoxifene also decreased non-HDL-C levels. Therefore, we believe that bazedoxifene improves lipid metabolism. Whether the LDL-C lowering and HDL-C increasing effects of SERMs also have an anti-arteriosclerotic effect has not been clearly answered. Raloxifene improves flow-mediated vasodilatation (FMD) and intima-media thickness (IMT) [27, 28]. Raloxifene also decreases inflammatory cytokines such as TNF- $\alpha$, IL-6, and MCP-1 that cause arteriosclerosis [29-31]. In large-scale clinical studies, however, raloxifene has not been reported to reduce cardiovascular or cerebrovascular events $[16,32]$. In the present study, arteriosclerosis indices such as hs-CRP and baPWV were not improved.

With respect to glucose metabolism, as previously reported with raloxifene [15], treatment with bazedoxifene in the present study produced no changes in glycemic control, insulin resistance, and insulin secretion in humans, and raloxifene has the report to suggest possibility to prevent the onset of diabetes in rats [33]. However, bazedoxifene did decrease adiponectin levels. Adiponectin is mainly affected by visceral fat mass and ucOC $[34,35]$. In particular, adiponectin decreases with an increase in visceral fat mass. The present study patients had no weight changes, so the decrease in adiponectin may have been associated with a decrease in the bone formation marker ucOC. In OC knockout mice, adiponectin is decreased, whereas in Esp-/- mice, which have high OC levels with opposite characteristics, the ucOC concentration compared to total $\mathrm{OC}$ is high, and adiponectin is increased. This suggests that ucOC may regulate adiponectin. It is suggested that SERMs secondarily decrease ucOC, and this may lead to a decrease in adiponectin.

The present study has a few limitations. First, this study had a small sample size and no control group. However, this study was a pilot study, and no information was available to calculate the sample size. The second limitation was the short study period. A period of 12 weeks was set in a manner similar to many studies of the evaluation of bone resorption markers [26]. However, it might be short in terms of the evaluation of the changes in bone formation and quality markers. Re-evaluation of bone formation markers after the administration of SERMs is recommended 24 weeks later [18], and a study reported that bone quality markers improved 24 weeks after the administration of SERMs [23]. Further studies may be needed with a study period longer than 12 weeks. 
Bazedoxifene tended to be more effective in preventing bone resorption in the $\mathrm{HbAlc} \geq 6.5 \%$ group than in the $\mathrm{HbA} 1 \mathrm{c}$ $<6.5 \%$ group. This suggests that the bone resorption improvement effects of bazedoxifene may differ depending on the degree of baseline glycemic control. Therefore, further studies on the effectiveness of bazedoxifene in T2DM patients with poor glycemic control should be conducted. In addition, in the bazedoxifene-responsive group based on TRACP-5b reduction, elevation of pentosidine tended to be prevented. Thus, pentosidine levels may be useful in assessing the bone quality improvement effects of bazedoxifene, and further studies in a larger number of patients would be desirable.

The present study, which was exploratory in nature, may be very valuable for constructing hypotheses leading to future studies and for establishing new treatments. However, no causal relationship between treatment with bazedoxifene and effectiveness can be demonstrated by the present study results alone. Large-scale comparative studies are needed for more confirmatory evidence [36, 37].

In conclusion, bazedoxifene may improve bone resorption markers and LDL-C levels without affecting glucose metabolism in postmenopausal patients with T2DM with good glycemic control.

\section{Acknowledgement}

This study was supported by a research fund from Pfizer Inc. Pfizer had no role in the conduct of the study or the analysis or interpretation of the data. Clinical Study Support, Inc. had roles in the study office, data management, and statistical analyses.

\section{Conflict of Interest}

Yasuo Terauchi received honoraria for lectures from MSD K.K.; Ono Pharmaceutical Co., Ltd; Nippon Boehringer Ingelheim Co., Ltd; Novartis Pharma K.K.; Takeda Pharmaceutical Co., Ltd; Mitsubishi Tanabe Pharma Corp.; Daiichi Sankyo Co., Ltd; Sanwa Kagaku Kenkyusho Co., Ltd; Kowa Pharmaceutical Co., Ltd; Novo Nordisk Pharma Ltd; Eli Lilly Japan K.K.; Sanofi K.K.; Shionogi \& Co., Ltd; Bayer Yakuhin, Ltd; and AstraZeneca K.K. and obtained research support from MSD K.K.; Ono Pharmaceutical Co., Ltd; Nippon Boehringer Ingelheim Co., Ltd; Novartis Pharma K.K.; Takeda Pharmaceutical Co., Ltd; Mitsubishi Tanabe Pharma Corp.; Daiichi Sankyo Co., Ltd; Sanwa Kagaku Kenkyusho Co., Ltd; Novo Nordisk Pharma Ltd; Eli Lilly Japan K.K.; Sanofi K.K.; Dainippon Sumitomo Pharma Co., Ltd; Shionogi \& Co., Ltd; Bayer Yakuhin, Ltd; Astellas Pharma, Inc.; Pfizer Japan, Inc.; and AstraZeneca K.K. Taishi Yoshii, Masayo Yamada, Taichi Minami, Tetsuji Tsunoda, Mayuko Sasaki, Yoshinobu Kondo and Shinobu Satoh declare that they have no conflict of interest.

\section{References}

1. Ferron M, Wei J, Yoshizawa T, Del Fattore A, DePinho
RA, Teti A, Ducy P, et al. Insulin signaling in osteoblasts integrates bone remodeling and energy metabolism. Cell. 2010;142(2):296-308.

2. Akune T, Ogata N, Hoshi K, Kubota N, Terauchi Y, Tobe $\mathrm{K}$, Takagi $\mathrm{H}$, et al. Insulin receptor substrate-2 maintains predominance of anabolic function over catabolic function of osteoblasts. J Cell Biol. 2002;159(1):147-156.

3. Vestergaard P. Discrepancies in bone mineral density and fracture risk in patients with type 1 and type 2 diabetes--a meta-analysis. Osteoporos Int. 2007;18(4):427-444.

4. Osteoporosis prevention, diagnosis, and therapy. JAMA. 2001;285(6):785-795.

5. Shiraki M, Kuroda T, Shiraki Y, Tanaka S, Higuchi T, Saito M. Urinary pentosidine and plasma homocysteine levels at baseline predict future fractures in osteoporosis patients under bisphosphonate treatment. J Bone Miner Metab. 2011;29(1):62-70.

6. Yamamoto M, Yamaguchi T, Yamauchi M, Yano S, Sugimoto T. Serum pentosidine levels are positively associated with the presence of vertebral fractures in postmenopausal women with type 2 diabetes. J Clin Endocrinol Metab. 2008;93(3):1013-1019.

7. Schwartz AV, Garnero P, Hillier TA, Sellmeyer DE, Strotmeyer ES, Feingold KR, Resnick HE, et al. Pentosidine and increased fracture risk in older adults with type 2 diabetes. J Clin Endocrinol Metab. 2009;94(7):2380-2386.

8. Fujii K, Kajiwara T, Kurosu H. Effect of vitamin B6 deficiency on the crosslink formation of collagen. FEBS Lett. 1979;97(1):193-195.

9. Liu G, Nellaiappan K, Kagan HM. Irreversible inhibition of lysyl oxidase by homocysteine thiolactone and its selenium and oxygen analogues. Implications for homocystinuria. J Biol Chem. 1997;272(51):32370-32377.

10. Saito M, Fujii K, Mori Y, Marumo K. Role of collagen enzymatic and glycation induced cross-links as a determinant of bone quality in spontaneously diabetic WBN/Kob rats. Osteoporos Int. 2006;17(10):1514-1523.

11. Mann V, Huber C, Kogianni G, Collins F, Noble B. The antioxidant effect of estrogen and Selective Estrogen Receptor Modulators in the inhibition of osteocyte apoptosis in vitro. Bone. 2007;40(3):674-684.

12. Gol M, Akan P, Dogan E, Karas C, Saygili U, Posaci C. Effects of estrogen, raloxifene, and hormone replacement therapy on serum C-reactive protein and homocysteine levels. Maturitas. 2006;53(3):252-259.

13. Christodoulakos G, Lambrinoudaki I, Panoulis C, Rizos D, Coutoukos J, Creatsas G. Effect of raloxifene, estrogen, and hormone replacement therapy on serum homocysteine levels in postmenopausal women. Fertil Steril. 2003;79(2):455-456.

14. Saito M, Marumo K, Soshi S, Kida Y, Ushiku C, Shinohara A. Raloxifene ameliorates detrimental enzymatic and nonenzymatic collagen cross-links and bone strength in rabbits with hyperhomocysteinemia. Osteoporos Int. 2010;21(4):655-666.

15. Cagnacci A, Paoletti AM, Zanni A, Arangino S, Ibba G, Orru M, Melis GB, et al. Raloxifene does not modify insulin sensitivity and glucose metabolism in postmenopausal women. J Clin Endocrinol Metab. 2002;87(9):4117-4121. 
16. Barrett-Connor E, Grady D, Sashegyi A, Anderson PW, Cox DA, Hoszowski K, Rautaharju P, et al. Raloxifene and cardiovascular events in osteoporotic postmenopausal women: four-year results from the MORE (Multiple Outcomes of Raloxifene Evaluation) randomized trial. JAMA. 2002;287(7):847-857.

17. Silverman SL, Christiansen C, Genant HK, Vukicevic S, Zanchetta JR, de Villiers TJ, Constantine GD, et al. Efficacy of bazedoxifene in reducing new vertebral fracture risk in postmenopausal women with osteoporosis: results from a 3-year, randomized, placebo-, and active-controlled clinical trial. J Bone Miner Res. 2008;23(12):1923-1934.

18. Nishizawa $Y$, Ohta $H$, Miura $M$, Inaba $M$, Ichimura S, Shiraki M, Takada J, et al. Guidelines for the use of bone metabolic markers in the diagnosis and treatment of osteoporosis (2012 edition). J Bone Miner Metab. 2013;31(1):1-15.

19. Nishizawa $Y$, Inaba $M$, Ishii $M$, Yamashita H, Miki T, Goto H, Yamada S, et al. Reference intervals of serum tartrate-resistant acid phosphatase type $5 \mathrm{~b}$ activity measured with a novel assay in Japanese subjects. J Bone Miner Metab. 2008;26(3):265-270.

20. Hannon RA, Clowes JA, Eagleton AC, Al Hadari A, Eastell R, Blumsohn A. Clinical performance of immunoreactive tartrate-resistant acid phosphatase isoform $5 \mathrm{~b}$ as a marker of bone resorption. Bone. 2004;34(1):187-194.

21. van Meurs JB, Dhonukshe-Rutten RA, Pluijm SM, van der Klift M, de Jonge R, Lindemans J, de Groot LC, et al. Homocysteine levels and the risk of osteoporotic fracture. N Engl J Med. 2004;350(20):2033-2041.

22. Shiraki M, Kuroda T, Tanaka S, Saito M, Fukunaga M, Nakamura T. Nonenzymatic collagen cross-links induced by glycoxidation (pentosidine) predicts vertebral fractures. J Bone Miner Metab. 2008;26(1):93-100.

23. Mori H, Okada Y, Kishikawa H, Inokuchi N, Sugimoto $\mathrm{H}$, Tanaka Y. Effects of raloxifene on lipid and bone metabolism in postmenopausal women with type 2 diabetes. J Bone Miner Metab. 2013;31(1):89-95.

24. Yamagishi S. Role of advanced glycation end products (AGEs) and receptor for AGEs (RAGE) in vascular damage in diabetes. Exp Gerontol. 2011;46(4):217-224.

25. Delmas PD, Bjarnason NH, Mitlak BH, Ravoux AC, Shah AS, Huster WJ, Draper M, et al. Effects of raloxifene on bone mineral density, serum cholesterol concentrations, and uterine endometrium in postmenopausal women. N Engl J Med. 1997;337(23):1641-1647.

26. Itabashi A, Yoh K, Chines AA, Miki T, Takada M, Sato H, Gorai I, et al. Effects of bazedoxifene on bone mineral density, bone turnover, and safety in postmenopausal
Japanese women with osteoporosis. J Bone Miner Res. 2011;26(3):519-529.

27. Sumino H, Ichikawa S, Kasama S, Takahashi T, Sakamoto H, Koizumi A, Kanai H, et al. Effects of raloxifene on brachial arterial endothelial function, carotid wall thickness, and arterial stiffness in osteoporotic postmenopausal women. Int Heart J. 2010;51(1):60-67.

28. Colacurci N, Fornaro F, Cobellis L, De Franciscis P, Torella M, Sepe E, Arciello A, et al. Raloxifene slows down the progression of intima-media thickness in postmenopausal women. Menopause. 2007;14(5):879-884.

29. Yasui T, Uemura H, Hyodo S, Yamada M, Yamamoto S, Maegawa M, Tsuchiya N, et al. Raloxifene reduces circulating levels of interleukin-7 and monocyte chemoattractant protein-1 in postmenopausal women. Atherosclerosis. 2009;204(2):471-475.

30. Gianni W, Ricci A, Gazzaniga P, Brama M, Pietropaolo M, Votano S, Patane F, et al. Raloxifene modulates interleukin-6 and tumor necrosis factor-alpha synthesis in vivo: results from a pilot clinical study. J Clin Endocrinol Metab. 2004;89(12):6097-6099.

31. Walsh BW, Cox DA, Sashegyi A, Dean RA, Tracy RP, Anderson PW. Role of tumor necrosis factor-alpha and interleukin-6 in the effects of hormone replacement therapy and raloxifene on $\mathrm{C}$-reactive protein in postmenopausal women. Am J Cardiol. 2001;88(7):825-828.

32. Barrett-Connor E, Mosca L, Collins P, Geiger MJ, Grady D, Kornitzer M, McNabb MA, et al. Effects of raloxifene on cardiovascular events and breast cancer in postmenopausal women. N Engl J Med. 2006;355(2):125-137.

33. Hill Gallant KM, Gallant MA, Brown DM, Sato AY, Williams JN, Burr DB. Raloxifene prevents skeletal fragility in adult female Zucker Diabetic Sprague-Dawley rats. PLoS One. 2014;9(9):e108262.

34. Lee NK, Sowa H, Hinoi E, Ferron M, Ahn JD, Confavreux C, Dacquin R, et al. Endocrine regulation of energy metabolism by the skeleton. Cell. 2007;130(3):456-469.

35. Yamauchi T, Kamon J, Waki H, Murakami K, Motojima $\mathrm{K}$, Komeda K, Ide T, et al. The mechanisms by which both heterozygous peroxisome proliferator-activated receptor gamma (PPARgamma) deficiency and PPARgamma agonist improve insulin resistance. J Biol Chem. 2001;276(44):41245-41254.

36. Albrecht J, Meves A, Bigby M. Case reports and case series from Lancet had significant impact on medical literature. J Clin Epidemiol. 2005;58(12):1227-1232.

37. Kooistra B, Dijkman B, Einhorn TA, Bhandari M. How to design a good case series. J Bone Joint Surg Am. 2009;91(Suppl 3):21-26. 